\title{
Implicit Nonlinear Wave Simulation with 1.08T DOF and 0.270T Unstructured Finite Elements to Enhance Comprehensive Earthquake Simulation
}

\author{
Tsuyoshi Ichimura $^{1}$, Kohei Fujita ${ }^{2}$, Pher Errol Balde Quinay $^{3}$, Lalith Maddegedara ${ }^{1}$, \\ Muneo Hori ${ }^{1,2}$, Seizo Tanaka ${ }^{4}$, Yoshihisa Shizawa ${ }^{5}$, Hiroshi Kobayashi ${ }^{5}$, and Kazuo Minami ${ }^{2}$ \\ ${ }^{1}$ Earthquake Research Institute and Department of Civil Engineering, The University of Tokyo \\ ${ }^{2}$ RIKEN Advanced Institute for Computational Science \\ ${ }^{3}$ Research Institute for Natural Hazards and Disaster Recovery, Niigata University \\ ${ }^{4}$ Faculty of Engineering, Information and Systems, University of Tsukuba \\ ${ }^{5}$ Research Organization for Information Science and Technology
}

\begin{abstract}
This paper presents a new heroic computing method for unstructured, low-order, finite-element, implicit nonlinear wave simulation: 1.97 PFLOPS (18.6\% of peak) was attained on the full $\mathrm{K}$ computer when solving a $1.08 \mathrm{~T}$ degrees-of-freedom (DOF) and $0.270 \mathrm{~T}-\mathrm{element}$ problem. This is 40.1 times more DOF and elements, a 2.68-fold improvement in peak performance, and 3.67 times faster in time-to-solution compared to the SC14 Gordon Bell finalist's state-ofthe-art simulation. The method scales up to the full $\mathrm{K}$ computer with 663,552 CPU cores with $96.6 \%$ sizeup efficiency, enabling solving of a $1.08 \mathrm{~T}$ DOF problem in $29.7 \mathrm{~s}$ per time step. Using such heroic computing, we solved a practical problem involving an area 23.7 times larger than the state-of-the-art, and conducted a comprehensive earthquake simulation by combining earthquake wave propagation analysis and evacuation analysis. Application at such scale is a groundbreaking accomplishment and is expected to change the quality of earthquake disaster estimation and contribute to society.
\end{abstract}

Categories: Time-to-solution, Scalability, Peak performance

\section{CONTRIBUtions OF SUPERCOMPUTERS TO REDUCING EARTHQUAKE DISASTERS}

\section{A. Overview and importance of the problem}

An earthquake can affect many people. The 2011 Tohoku Earthquake in Japan killed 20,000 and more than 200,000 people are still in temporary housing. The loss of lives, damage to the economy, and catastrophic damage are fresh in our memory. This damage occurred in Japan, a country that leads the world in earthquake disaster mitigation, and there are concerns over similar disasters in earthquake-prone mega-cities such as Los Angeles, San Francisco, and Tokyo. Reliable earthquake disaster estimation plays an important role in mitigating such disasters. Physics-based comprehensive earthquake simulation is the only way to make reliable estimations of such infrequent and untestable events, and is

Permission to make digital or hard copies of all or part of this work for personal or classroom use is granted without fee provided that copies are not made or distributed for profit or commercial advantage and that copies bear this notice and the full citation on the first page. Copyrights for components of this work owned by others than ACM must be honored. Abstracting with credit is permitted. To copy otherwise, or republish, to post on servers or to redistribute to lists, requires prior specific permission and/or a fee. Request permissions from Permissions@acm.org < mailto:Permissions@acm.org $>$.SC '15, November 15-20, 2015, Austin, TX, USA (C)2015 ACM. ISBN 978-14503-3723-6/15/11 \$15.00 DOI: http://dx.doi.org/10.1145/2807591.2807674

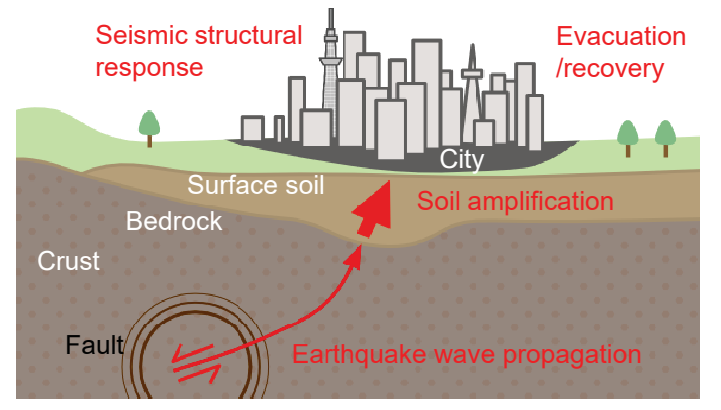

Fig. 1. An urban earthquake disaster consists of earthquake wave propagation, soil amplification, the seismic structural response, and evacuation/recovery. The contribution of supercomputing is eagerly anticipated, because physicsbased comprehensive earthquake simulation including these processes requires huge computational cost.

a key to disaster mitigation. However, it is still difficult to realize such simulations due to their huge computation costs. The contributions of supercomputing to this field are eagerly anticipated.

\section{B. State of the art and achievements of this paper}

An urban earthquake disaster involves the propagation of seismic waves caused by fault rupture through the Earth's crust (earthquake wave propagation), nonlinear wave propagation near the surface due to strong material nonlinearity (soil amplification), the response of buildings due to ground shaking (structural response), and the response of humans to these phenomena (evacuation and recovery); see Fig. 1. Evaluations based on comprehensive physics-based simulations of these processes should improve the reliability of urban earthquake disaster estimation, but this has not been accomplished due to the huge computing cost. Therefore, statistical methods relating earthquake source intensity to ground motion intensity, or ground motion intensity to building damage [1], [2], [3] are typically used, rather than physics-based evaluations. To achieve a breakthrough in earthquake disaster estimation, we introduce a comprehensive physics-based earthquake simulation of all four disaster processes using massive computation. This is the first analysis of this kind, and is an important step in the use of supercomputing to reduce earthquake disasters.

Here, we present a comprehensive earthquake simulation of 


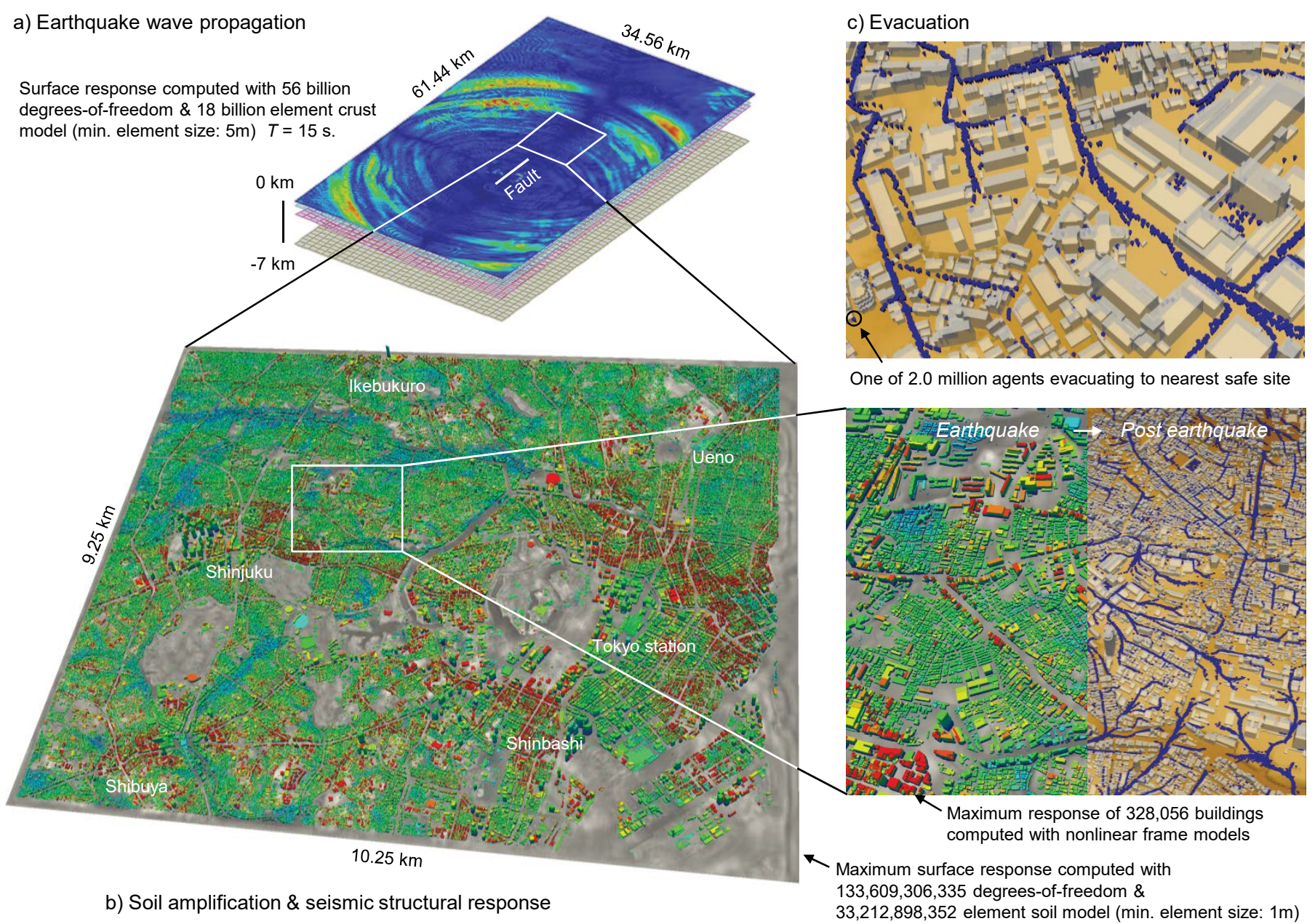

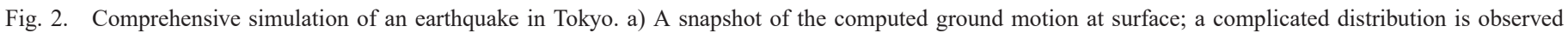

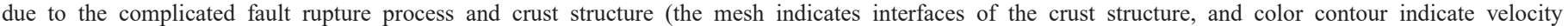

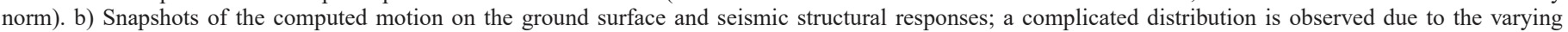

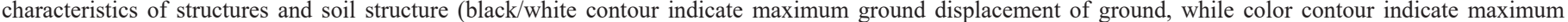

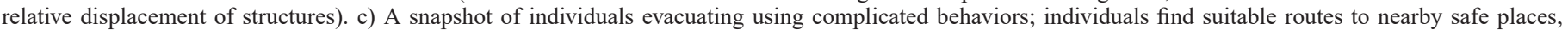
considering many interaction types in a complex urban environment.

an actual city that was performed on the $\mathrm{K}$ computer (details of the $\mathrm{K}$ computer [4] are summarized in the next section), together with state-of-the-art supercomputing. We target the center of Tokyo in Japan, an area measuring $10.25 \times 9.25$ $\mathrm{km}$ with 328,056 structures, which includes financial, business and commercial centers, government offices, and densely populated residential districts, and simulate the response of the city and evacuation of people in an assumed Tokyo earthquake. The earthquake wave propagation simulation and evacuation/recovery simulation shown below compare favorably to the current state of the art, while the soil amplification simulation in this comprehensive simulation easily surpasses the current state of the art by fully utilizing the power of the entire supercomputer system. This paper includes an innovative nonlinear wave analysis for soil amplification, which enables heroic computing in a comprehensive earthquake simulation. This innovation is the main achievement of this paper and the details are presented in Section II.

1) Earthquake wave propagation: With the accumulation of crust data and developments in supercomputing, many studies have estimated ground motion based on three-dimensional
(3D) linear wave propagation. Computations analyzing fault rupture and wave propagation over huge areas with high resolution have huge computational costs and have been a hot topic at Supercomputing Conferences (SCs) (e.g., [5], [6], [7], [8]). The current state of the art in this field is typified by SeisSol [9], which is based on the discontinuous Galerkin method with high-order elements. In their SC14 Gordon Bell finalist paper, SeisSol accomplished a practical simulation with 191 million tetrahedral elements and 96 billion degrees-offreedom (DOF), corresponding to $10-\mathrm{Hz}$ accuracy, which is important for engineering purposes. Here, we constructed a low-order unstructured/structured hybrid finite element model (an 18 billion first-order finite element, 56 billion DOF model, with a minimum element size of $5 \mathrm{~m}$ ), targeting an area 34.56 $\times 61.44 \times 60 \mathrm{~km}$, based on the crust model of [10] and source model of [11], and solved a 31.2-s linear earthquake propagation problem using implicit time integration. Because the Earth's crust has large material heterogeneity by nature, many elements are needed for discretization: we use 94 times more elements than the state of the art, using 36,864 compute nodes $\times 8$ OpenMP threads $=294,912 \mathrm{CPU}$ cores of the 
$\mathrm{K}$ computer for this problem. Fig. 2a) shows a snapshot of the computed ground motion caused by the assumed Tokyo earthquake. Here, we use enhanced code of [12]; although there is room left for further performance tuning, it is still comparable to the state of the art. However, its computation scale is still small compared to the nonlinear wave analysis involved in soil amplification, which we show next.

2) Soil amplification and seismic structural response: With the accumulation of urban spatial data, studies are beginning to examine soil amplification and seismic structural response simulations using physics models of cities. Owing to the huge computational cost, there has been little research on this topic (e.g., [13], [14], [15]), although it is expected to be a frontier in supercomputing. The soil amplification process includes the localized nonlinear response of ground due to its complex geometry and high material heterogeneity. Loworder unstructured 3D nonlinear wave simulation is used to model these phenomena, leading to huge computational costs. One current state of the art in nonlinear wave simulation is the SC14 Gordon Bell prize finalist GAMERA [16], which computed the seismic response of a $2.0 \times 2.0-\mathrm{km}$ area of an actual city with 13,275 structures. This simulation used 36,864 compute nodes $\times 8$ OpenMP threads $=294,912$ CPU cores of the $\mathrm{K}$ computer to compute the nonlinear wave problem for a $2.0 \times 2.0 \times 0.1-\mathrm{km}$ area with 10.7 billion DOF and 2.67 billion finite elements. In comparison, the seismic response analysis for an assumed Tokyo earthquake must simulate a $10.25 \times 9.25 \times 0.24-\mathrm{km}$ area with 0.133 tera-DOF and 33 billion elements; this is 23.7 times larger in area and 12.4 times larger in DOF than the state of the art computation at $\mathrm{SC} 14$. Our model includes a complex three-layered ground structure constructed from the 5-m digital elevation map of the Geographic Information Authority of Japan, the Digital Soil Map [17], and material properties obtained from the National Research Institute for Earth Science and Disaster Prevention [18], and is discretized with an element size sufficiently small for convergence of the acceleration response, even when the ground is softened due to nonlinearity. Using this finite element model, we input the main part of the computed wave of the earthquake wave propagation simulation shown in the last section, and compute the nonlinear wave equation $(6,600$ time steps with a $0.002 \mathrm{~s}$ time increment) using the full $\mathrm{K}$ computer $(82,944$ nodes $\times 8 \mathrm{CPU}$ cores $=663,552 \mathrm{CPU}$ cores $)$. Next, we compute the response of 328,056 structures located inside the domain using the computed ground motion. Fig. 2b) shows snapshots of the computed ground motion on the ground surface and the seismic structural responses. We can see that not all of the structures show a marked response, even in areas with large ground motion. Such a complex response in a city can be computed only by combining a high-resolution 3D nonlinear wave simulation and simulating the responses of many buildings in the city.

3) Evacuation/recovery: To guarantee the safety of the residents, it is essential to ensure that all can reach safe evacuation areas within a reasonable time. After a major earthquake, some roads can be rendered unusable by debris, fires, and the like. One of the best approaches to addressing such needs by quantitatively comparing strategies to speed and facilitate the evacuation process is the mass evacuation simulator based on Multi Agent Systems. The state of the art is [19], which simulated the evacuation of 200,000 agents in a
$15 \times 7.5-\mathrm{km}$ area. In comparison, because our target area has a high population density of 18,000 people $/ \mathrm{km}^{2}$ and limited open spaces, mass evacuation simulation using the Multi Agent System would be challenging. Our evacuation simulation is parallelized using the Message Passing Interface (MPI), and techniques such as dynamic load balancing are used to maintain scalability [20], [21], [22]. We simulate the evacuation of 2,000,000 agents in this area, using 2,048 CPU cores of the Kcomputer. Fig. 2c) shows a snapshot of the agents evacuating to nearby safe places. An evacuation simulation that considers many types of interaction in a complex urban environment is conducted and is expected to provide valuable information concerning the safety of residents.

\section{DETAILS OF HEROIC COMPUTING}

New heroic computing of soil amplification is needed to accomplish a comprehensive earthquake simulation with the physics-based modeling shown in Section I. This practical problem should be solved by enabling low-order, unstructured finite element simulation with 1.0 TDOF with 20\% peak performance on the full $\mathrm{K}$ computer system. However, this requires innovations corresponding to a 40 -fold increase in DOF and a 2.5-fold higher peak performance compared to the state of the art (27 billion DOF problem solved with GAMERA in $\mathrm{SC} 14$ [16]).

\section{A. Target equation}

The soil amplification simulation computes the nonlinear propagation of seismic waves in a ground structure model. Although the ground structure is typically modeled as a layered structure, the layer thickness can change $10^{-1 \sim 1} \mathrm{~m}$ in a $10^{0 \sim 1}$ $\mathrm{m}$ area, leading to very complex geometry. This leads to nonuniform wave amplification and causes local soil softening, which propagates through the soil and changes the material heterogeneity dynamically during the process. Although initially uniform, material heterogeneity occurs and propagates in each soil layer, leading to a dynamically changing and highly heterogeneous problem. To solve this problem, we use a nonlinear dynamic 3D finite element method using very small low-order unstructured solid elements. This method analytically satisfies the traction-free condition at the surface and is suitable for modeling complex geometry and highly heterogeneous material.

$$
\begin{aligned}
& \left(\frac{4}{d t^{2}} \mathbf{M}+\frac{2}{d t} \mathbf{C}^{n}+\mathbf{K}^{n}\right) \delta \mathbf{u}^{n}= \\
& \mathbf{F}^{n}-\mathbf{Q}^{n-1}+\mathbf{C}^{n} \mathbf{v}^{n-1}+\mathbf{M}\left(\mathbf{a}^{n-1}+\frac{4}{d t} \mathbf{v}^{n-1}\right),
\end{aligned}
$$

with

$$
\left\{\begin{array}{l}
\mathbf{Q}^{n}=\mathbf{Q}^{n-1}+\mathbf{K}^{n} \delta \mathbf{u}^{n}, \\
\mathbf{u}^{n}=\mathbf{u}^{n-1}+\delta \mathbf{u}^{n}, \\
\mathbf{v}^{n}=-\mathbf{v}^{n-1}+\frac{2}{d t} \delta \mathbf{u}^{n}, \\
\mathbf{a}^{n}=-\mathbf{a}^{n-1}-\frac{4}{d t} \mathbf{v}^{n-1}+\frac{4}{d t^{2}} \delta \mathbf{u}^{n} .
\end{array}\right.
$$

Here, $\delta \mathbf{u}, \mathbf{u}, \mathbf{v}, \mathbf{a}$, and $\mathbf{F}$ indicate the incremental displacement, displacement, velocity, acceleration, and external force, respectively; $\mathbf{M}, \mathbf{C}$, and $\mathbf{K}$ are the consistent mass matrix, Rayleigh damping matrix, and stiffness matrix, respectively; and $d t$ and $n$ indicate the time step increment and time step number. Small elements are sometimes generated when modeling the precise geometry of complex geometry problems with solid elements. 
Consequently, using explicit time integration (e.g., a central difference scheme) leads to very small time increments to satisfy the Courant condition. To avoid the huge computation cost of small time increments, we use the Newmark- $\beta$ method (implicit time integration). We use second-order tetrahedral elements, with a half-infinite absorbing boundary condition for the bottom and four sides of the domain boundary. We use a modified RO model [23] with the Masing rule [24] as a nonlinear constitutive model of soil. To solve the dynamic 3D nonlinear ground displacement $\mathbf{u}^{n}$, we repeat the following process:

1) Read boundary condition;

2) Evaluate $\mathbf{C}^{n}$ and $\mathbf{K}^{n}$ using the strain computed at time step $n-1$;

3) Solve Eq. (1) to get $\delta \mathbf{u}^{n}$;

4) Update the values in Eq. (2) using $\delta \mathbf{u}^{n}$;

5) Output results

Almost all of the computation cost lies in solving Eq. (1), making it necessary to develop a solver suitable for nonlinear waves.

\section{B. Key ideas and innovations}

To obtain convergence of the acceleration waveform and the response of buildings for a megacity problem (100 square $\mathrm{km}$ ), it is necessary to solve nonlinear wave propagation with a 100 billion DOF 3D finite element model for $10 \mathrm{~K}$ time steps. In other words, the problem involves solving a 100billion DOF matrix equation (Eq. (1)) using many computation nodes for each of the $10 \mathrm{~K}$ time steps. The matrix component changes every time step due to nonlinearity. Rapid analysis is desirable due to time constraints and efficient use of computing resources. When using the $\mathrm{K}$ computer, we need to solve $10 \mathrm{~K}$ time steps in $8 \mathrm{~h}$. Because this is equivalent to $2.88 \mathrm{~s}$ per time step, we need a very fast solver. We use an iterative solver, as the DOF of the problem becomes large compared to the computing resources. Developing an iterative solver suitable for the matrix property is required for fast analyses. Many methods have been proposed for low-order unstructured finite element methods for solid problems (e.g., [25], [26]), and some have been presented at past SCs (e.g., [27]). For example, [25] presents a fast solver based on a balancing domain decomposition method [28]-type preconditioner, which could solve a 140 M-DOF problem in $577 \mathrm{~s}$ (3.88 TFLOPS, $24.26 \%$ of peak) with 3.05 TB memory and 2,048 CPUs on the Earth Simulator [29]. These researchers sought to reduce the computation time by using a preconditioner with a high computation cost to improve the poor convergency of the target problem. Although a sophisticated preconditioner with smart program tuning can reduce the computation cost and resolve the poor convergency of the target problem, our problem needed a much faster solver. Although the nonlinear wave propagation problem is challenging in that we need to solve many time steps with matrix components changing in every time step, the matrix property is not as bad due to inertia terms. These properties are the key to a fast solver. Potentially, the preconditioned conjugate gradient (PCG) method using a block Jacobi preconditioner [34] is such a solver, but the number of iterations tends to increase rapidly when solving problems with poor convergence characteristics. GAMERA [16] was developed as a faster solver than PCG, but its performance is too poor for our purpose. Here, we improve GAMERA to solve a 1TDOF problem, which is the edge of the low-order finite element problem.

Let us review the history of earthquake wave propagation analysis (linear wave), which deals with wave propagation phenomena similar to those targeted in our nonlinear wave propagation problem. With the need for high-resolution wave propagation analysis in large target domains, the wave propagation problem becomes a huge problem and has been a touchstone of massive computing. Much effort has been directed toward the development of wave propagation analysis, which gives insight into the development of nonlinear wave analysis. A structured, finite difference scheme was the first used to study practical wave propagation in the crust, due to its low computational cost and efficient memory access. However, the effect of the geometry of crust structure in earthquake propagation is large, and so an unstructured finite element method capable of modeling geometry precisely was developed (e.g., [30]). Although unstructured finite element methods involve huge costs due to the large computation and memory access cost and the effort required for modeling a finite element mesh, research based on the finite-element family is increasing due to its advantages for precise geometry modeling with highly accurate numerical results (e.g., [7], [9]). In an attempt to reduce the cost of finite element methods, a structured finite element method using an octree structure was developed (e.g., [5]). This method can also be considered an enhancement of structured finite difference methods, because it is capable of reducing the number of elements due to multi-resolution modeling. Compared to the unstructured finite element method, the octree method reduces computation and memory access costs and it is easier to construct finite element models. However, it still has drawbacks in terms of geometry modeling. Alternatively, finite element methods that use hybrid unstructured/structured elements (unstructured tetrahedral elements for domains with a complex geometry, and structured hexahedral elements for uniform domains) have been developed (e.g., [31], [32]). With these insights, we can see how GAMERA should evolve, by reducing computation and memory access costs using octreelike restrictions for uniform domains, while using unstructured tetrahedral elements for domains with complex geometry.

Here, we enhance GAMERA based on these insights; we make a fast solver capable of many-time-step nonlinear wave analysis for a multi-compute node environment by reducing computing costs, using effective preconditioning with small computing costs and a small memory footprint, and reducing communication for better scalability. Algorithms 1 and 2 are used to solve $\delta \mathbf{u}^{n}$ of $n$-th time steps, by combining methods a) to d) below. Hereafter, we refer to this solver as GOJIRA, as an abbreviation of the components used ${ }^{1}$.

a) Adaptive conjugate gradient method [33] with a mixed precision operation and multigrid method [34]: Typically, the inverse of a matrix close to the matrix of the matrix equation is used as the preconditioner of a conjugate gradient iteration. The adaptive conjugate gradient method uses conjugate gradient iteration to solve the matrix equation roughly, instead of this preconditioner. The original conjugate gradient iteration is

\footnotetext{
${ }^{1}$ Geometrically cOnstrained conJugate gradIent solve $\mathbf{R}$ with multi-grid and mixed-precision Arithmetic. Together with GAMERA, GOJIRA is an imaginary Japanese guardian, defending humanity from three-headed monsters.
} 


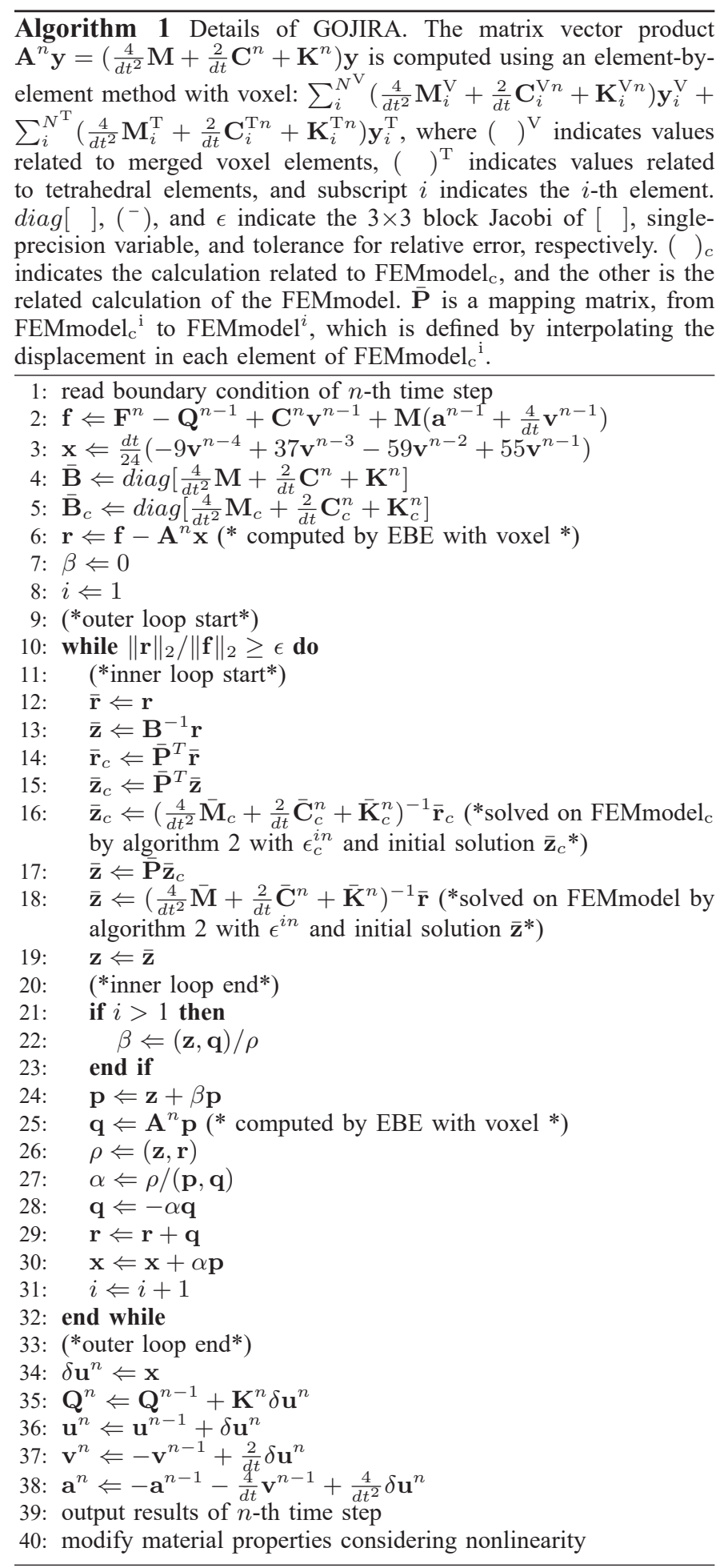

called the outer loop, while the conjugate gradient iteration in the preconditioner is called the inner loop. Because we need only to solve the matrix equation roughly in the inner loop, there is room for improvement. First, we compute the inner loop in single precision as we can reduce precision in the inner loop. We can expect to make computation faster by increasing the peak performance of some hardware, reducing memory transfer sizes, increasing apparent cache capacity, and reducing the data size of MPI communications. Next, we use multigrid
Algorithm 2 Preconditioned conjugate gradient method with reduced memory access. This is used to roughly solve $\mathbf{z}=\left(\frac{4}{d t^{2}} \mathbf{M}+\right.$ $\left.\frac{2}{d t} \mathbf{C}^{n}+\mathbf{K}^{n}\right)^{-1} \mathbf{r}$ and $\mathbf{z}_{c}=\left(\frac{4}{d t^{2}} \mathbf{M}_{c}+\frac{2}{d t} \mathbf{C}_{c}^{n}+\mathbf{K}_{c}^{n}\right)^{-1} \mathbf{r}_{c}$ in Algorithm 1. The matrix vector product $\mathbf{A}^{n} \mathbf{y}=\left(\frac{4}{d t^{2}} \mathbf{M}+\frac{2}{d t} \mathbf{C}^{n}+\right.$ $\left.\mathbf{K}^{n}\right) \mathbf{y}$ is computed using an element-by-element method with voxel: $\sum_{i}^{N^{\mathrm{V}}}\left(\frac{4}{d t^{2}} \mathbf{M}_{i}^{\mathrm{V}}+\frac{2}{d t} \mathbf{C}_{i}^{\mathrm{V} n}+\mathbf{K}_{i}^{\mathrm{V} n}\right) \mathbf{y}_{i}^{\mathrm{V}}+\sum_{i}^{N^{\mathrm{T}}}\left(\frac{4}{d t^{2}} \mathbf{M}_{i}^{\mathrm{T}}+\frac{2}{d t} \mathbf{C}_{i}^{\mathrm{T} n}+\right.$ $\left.\mathbf{K}_{i}^{\mathrm{T} n}\right) \mathbf{y}_{i}^{\mathrm{T}}$, where ()$^{\mathrm{V}}$ indicates values related to merged voxel elements, ()$^{\mathrm{T}}$ indicates values related to tetrahedral elements, and the subscript $i$ indicates the $i$-th element. $\mathbf{B}$ and $\epsilon^{i n}$ are a block Jacobi matrix and the tolerance for the relative error, respectively. All of the calculations are made using single-precision variables. The computation in loop 3 is merged into one DO loop, which reduces the total memory access from 13 streams in the naive implementation to 8 streams per $\mathrm{CG}$ iteration.

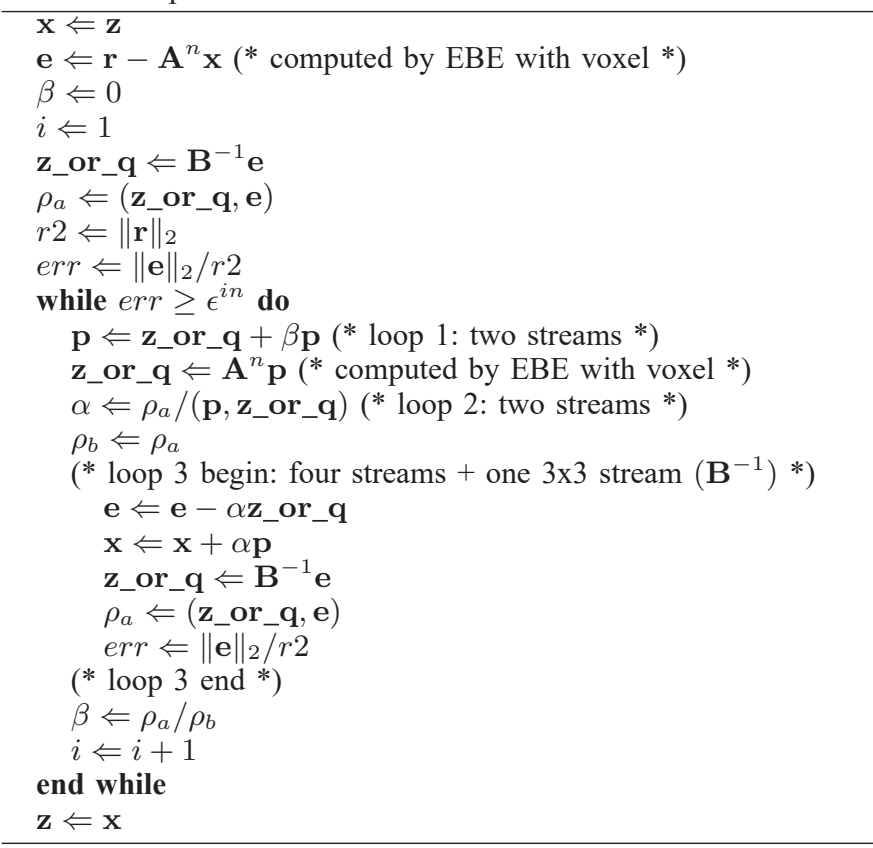

to reduce computation. Here, we use another model equivalent to the original problem, albeit one with a coarser resolution. Hereafter, we refer to the original model as FEMmodel and the coarsened model as FEMmodel $_{\mathrm{C}}$. In the inner loop, we first use FEMmodel $_{\mathrm{c}}$ to solve the problem roughly, and use this as an initial solution for the FEMmodel, to reduce the computation cost of the preconditioner. We can reduce the number of iterations when solving the FEMmodel roughly, and can also reduce floating-point operations by improving the convergence characteristics. Although we also need to compute FEMmodel $_{\mathrm{c}}$, it has fewer DOF than the original problem, and fewer finite element nodes to be communicated between MPI processes. Consequently, the computation cost and communication size become smaller than with the FEMmodel, markedly reducing the cost of the inner loop. As a result, multigrid can reduce computation. Because the communication involved in mapping values between the fine and coarse grids can be a bottleneck when running on massively parallel computers, we use a first-order tetrahedral element model for FEMmodel $\mathrm{C}_{\mathrm{C}}$, which is generated by removing the edge nodes of the secondorder tetrahedral element model used for FEMmodel, and distribute the same parts of the sub-models to compute nodes. This enables mapping between FEMmodel and FEMmodel $_{\mathrm{C}}$ with small amount of communication. 
b) Element-by-element method [35]: Because the DOF per compute node is large, allocating and updating matrices $\mathbf{K}$ or $\mathbf{M}$ becomes costly and it sometimes becomes impossible to store data within memory capacity. Therefore, we compute the matrix vector products $\mathbf{K u}$ and $\mathbf{M u}$ of the FEMmodel and $\mathrm{FEMmodel}_{\mathrm{c}}$ by using an element-by-element (EBE) method instead of storing the entire $\mathbf{K}$ and $\mathbf{M}$ matrices in memory. Using orthogonal functions for bases of stress (displacementstress mixed formulation of finite element method [36]), we transform $\mathbf{K u}$ analytically into $\sum_{i} \mathbf{N}_{\mathbf{b} i}^{T} \mathbf{D} \mathbf{N}_{\mathbf{b} i} \mathbf{u}$, and compute the matrix vector product in the order of $\sum_{i} \mathbf{N}_{\mathbf{b} i}^{T}\left(\mathbf{D}\left(\mathbf{N}_{\mathbf{b} i} \mathbf{u}\right)\right)$. Here, $\mathbf{D}$ is the elasticity matrix and $\mathbf{N}_{\mathbf{b} i}$ is the equivalent mode. Because $\mathbf{N}_{\mathbf{b} i}$ and $\mathbf{D}$ consist of many zero components, we compute only necessary components by explicitly expanding the computation into scalar expressions, which eliminates unnecessary computation. We also compute $\mathrm{Mu}$ element by element, and add the results into the global left-hand-side vector.

c) "Structuring" unstructured mesh: When using the highly efficient algorithm involving a) and b), the communication cost becomes negligible and the reduction in computation time and improvement in load balancing becomes the key to speeding up the solver. Considering computation, most of the time is spent on the EBE computation of the inner fine, inner coarse, and outer loops. Although the computation cost is large, it involves indirect list accesses and the recurrence of data. EBE computation suitable for existing CPUs with multi-core and single instruction multiple data (SIMD) units is important for speedup. In modern computers, computation arranged in a structured manner leads to efficient computation, and structured geometry leads to a reduction in computation. Although an unstructured mesh inherently involves unstructured computation, we extract uniform parts inside the complex geometry, and compute these parts using structured computation. Here, we generate a mesh with multi-resolution voxel and tetrahedra, and further decompose each voxel with six tetrahedra; see Fig. 3. Although the resulting mesh is an unstructured mesh consisting of second-order tetrahedral elements, uniform parts inside the domain are structured; these are decomposed with six types of elements with fixed geometry. Because we can skip parts regarding geometry in the computation of $\mathbf{K u}$, we can reduce the number of floating point operations of EBE computation by $1 / 2.12$ compared to normal secondorder tetrahedral elements. We can also reduce the number of list accesses from 60 (10 nodes per second-order tetrahedral element $\times 6$ elements) to 27 when computing six elements together in one voxel. The same can be applied to first-order tetrahedral elements; there is a $1 / 3.55$ reduction of floating point operations for the EBE computation, and reduction in list accesses from 24 ( 4 nodes $\times 6$ ) to 8 . Because the computations and list accesses are reduced, we can expect to reduce the time-to-solution and improve the efficiency of computation. By buffering the nodal data of elements, we can make the data structure into contiguous access without recurrences, which is suitable for SIMD computation.

d) Steady contiguous memory access computation: With the very fast matrix vector computation enabled by c), even contiguous memory access computation (i.e., vector addition, inner product) becomes apparent in the total computation time. With such computation, the penalty caused by cache thrashing a)

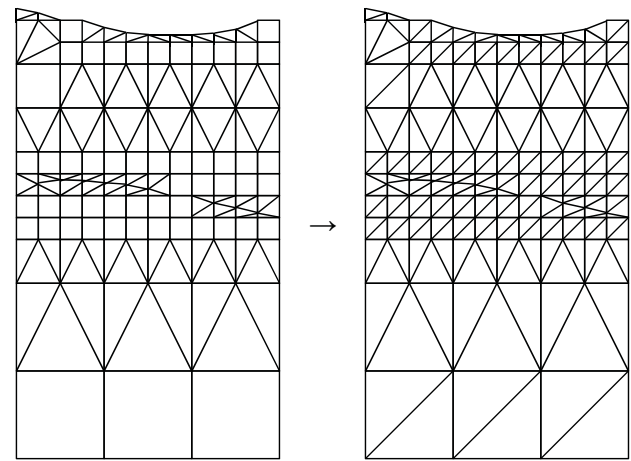

b)

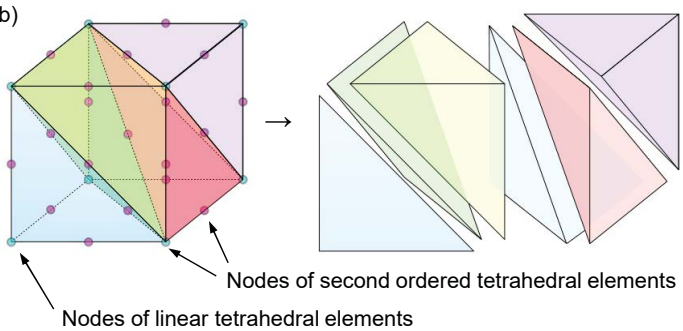

Fig. 3. "Structuring" unstructured mesh. a) A hybrid mesh of multi-resolution voxel mesh and unstructured tetrahedral mesh is converted to an unstructured tetrahedral mesh combined with a tetrahedral mesh with fixed geometry. b) Decomposition of voxel to tetrahedra. Each voxel is converted to six tetrahedral elements with fixed geometry.

becomes large; it can take several times longer to compute than cases without thrashing. Although it is rare to have thrashing occur for a specific array length, many partitions with different array lengths are generated when using graph (mesh) partitioning libraries such as METIS [37]. In such cases, it is highly probable that some nodes will have cache thrashing when analyzing in a massively parallel environment, which would lead to load unbalance. Here, we allocate arrays with the same length, and use only the necessary parts of them, so that all nodes have similar memory alignments. We also exchange the order of DO loops inside the standard PCG, and merge these loops to reduce memory access from 13 streams to 8 (see Algorithm 2). By combining this with single-precision arithmetic, we can reduce memory access to $8 /(13 \times 2)=1 / 3.25$ that of the original PCG solver.

GOJIRA uses the inner loop for preconditioning and the outer loop for the normal conjugate gradient iteration using a) and b); efficient preconditioning is applied with a small memory requirement, fast convergence, and reduced computation, while the outer loop attains small-memory-requirement computation. In addition, c) and d) enable efficient memory access and a reduction in computation by applying constraints in the arithmetic space of a) and b). The computation cost of a single outer loop is similar to that of one PCG loop, but the number of iterations becomes small due to the effective preconditioning by the inner loop. Because most computation time is spent in the fast inner loops, the computation time of the entire solver decreases. GOJIRA should be effective on hardware that uses fast single-precision arithmetic (e.g., an Intel CPU or GPU) or systems with slow communication interconnects, but fast computation is expected on systems like the $\mathrm{K}$ computer, which have the same peak performance regardless of whether single or double precision is used, due 
to faster data access. The finite element models are generated as in [16], and partitioned using METIS. We also use the Adams-Bashforth method [38] to guess the initial solution of an iterative solver when solving $\delta \mathbf{u}^{n}$.

\section{Performance measurement}

1) System and environment in which performance was measured: Although GOJIRA is more suitable for environments with fast single-precision arithmetic, we used the K computer at RIKEN, Advanced Institute for Computational Science [4], to measure performance as we can use many compute nodes and solve larger problems. The $\mathrm{K}$ computer is a massively parallel supercomputer consisting of 82,944 compute nodes, each with a single SPARC64 ${ }^{\mathrm{TM}}$ VIIIfx CPU. Each of the eight cores of the SPARC CPUs has two sets of fused multiplyadd (FMA) SIMD units of length 2. This leads to FMA (two operations $) \times$ SIMD length $2 \times 2$ sets $=8$ operations per clock. Consequently, the peak performance of each CPU is 8 operations $\times 8$ cores $\times 2 \mathrm{GHz}=128$ giga-FLOPS (GFLOPS). The SIMD length is 2 for both single- and double-precision arithmetic, so that the peak performance of single-precision arithmetic is also 128 GFLOPS. Each core has two $32 \mathrm{~KB}$ twoway L1 caches, each for data and instructions, and the cores share a 6 MB 12-way L2 cache (shared with data/instruction). Each node has 16 GB of DDR3-SDRAM memory, with a peak memory bandwidth of $64 \mathrm{~GB} / \mathrm{s}$. Tofu, a six-dimensional interconnection network, is used for communication between nodes [39]. Each node can communicate in four directions simultaneously with $5 \mathrm{~GB} / \mathrm{s}$ throughput in each direction. An OpenMPI [40]-based MPI library optimized for the Tofu network is used.

2) Problem setting: We compared the effectiveness of GOJIRA to those of GAMERA and PCGE. GAMERA is a solver that was developed in an SC14 paper, and was used to solve a 27 billion DOF problem [16]. PCGE is a preconditioned conjugate gradient solver with EBE, which was referred to as a standard method in an SC14 paper [16]. GAMERA and PCGE were improved for solving large-scale problems. We use $\epsilon=10^{-8}$ for the relative error of the outer loop, and $\epsilon^{i n}=0.25, \epsilon_{c}^{i n}=0.5$ for the inner loops. Here, we assign the partitioned models FEMmodel ${ }^{i}$ and FEMmodel ${ }_{\mathrm{C}}{ }^{\mathrm{i}}$ to each node and compute each partition using OpenMP; we use $8 \times m$ CPU cores when using $m$ compute nodes ( $m$ MPI processes) of the $\mathrm{K}$ computer.

We made a two-layered finite element model mimicking actual ground, and measured the performance of nonlinear ground motion analysis by inputting a wave uniformly from the bottom of the model. The model has periodical geometry for use in weak scaling measurements; see Fig. 4 for the model and its material properties. The first layer is a soft layer that behaves nonlinearly under strong inputs, and the second layer is a hard layer mimicking engineering bedrock. The model has bumps between the layers. The model is copied in the $x$ and $y$ directions when making larger models. The models are discretized with a sufficiently small element size (minimum element size $0.444 \mathrm{~m}$ ) for convergence in the acceleration response, even if the soil is softened due to nonlinearity. The major part of the Kobe wave [41] $(d t=0.002 \mathrm{~s})$ observed during the 1995 Southern Hyogo Earthquake is used as input. Table I shows the properties of the weak scaling model

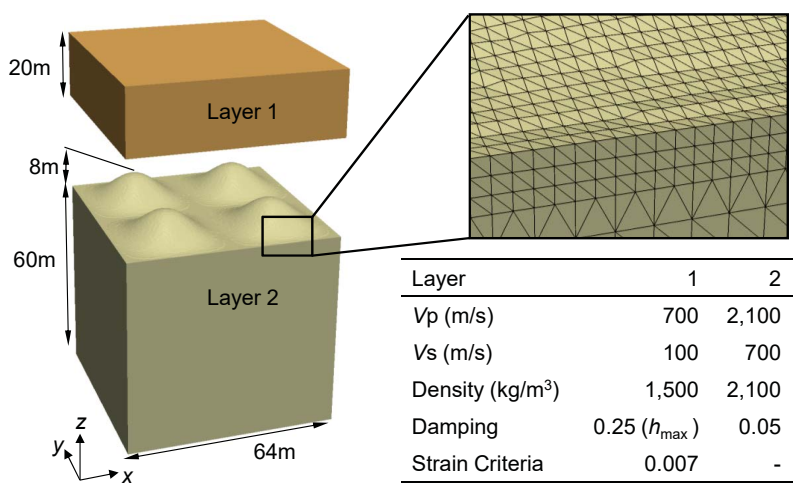

Fig. 4. Ground model for measuring performance. The model is duplicated in the $x$ and $y$ directions to make large-scale models with periodical geometry.

sets and strong scaling model set. Although the problem is periodical, the model is partitioned by METIS and the model sets are expected to have load balancing and communication characteristics similar to those of a practical problem. Here, we solve 3 steps for the weak scaling (model set A) model set and 25 steps for the weak scaling (model set B) and strong scaling (model set $\mathrm{C}$ ) model sets. Although the convergence characteristics change with the DOF as matrix equations are solved, the model sets are made with periodical settings so that the models have similar convergence characteristics regardless of DOF. In fact, the iterations required for convergence of the PCGE solver are almost the same as that required for all of the models in each model set. We can see that the model sets are suitable for measuring weak scalability.

3) Weak scaling up to $1 T$ DOF on full system: First, we measure weak scalability by fixing the problem size per node to 13.1 MDOF and increasing the number of nodes. Fig. 5 shows the elapsed time for the model set. Outer, inner fine, and inner coarse indicate the outer loop, line 18 of Algorithm 1, and line 16 of Algorithm 1, respectively. The numbers in red/green/blue indicate the number of loops required for convergence. We can see that the number of loops is similar among the models; the model set is suitable for measuring weak scalability. The smallest model, A-1, with 15.1 billion DOF, is solved in 86.2 s with 28.3 TFLOPS (19.2\% of peak) when using 9,216 CPU cores $(1,152$ nodes $\times 8 \mathrm{CPU}$ cores $)$, while the largest model, A-7, with 1.08 trillion DOF, is solved in $89.2 \mathrm{~s}$ with 1.97 PFLOPS (18.6\% of peak) when using the full K computer system with $663,552 \mathrm{CPU}$ cores $(82,944$ nodes $\times 8 \mathrm{CPU}$ cores). The size-up scalability up to the full system is $96.6 \%$; we see that fast analysis is enabled with excellent size-up scalability. In comparison, the elapsed time when using GAMERA is $328 \mathrm{~s}(8.60 \%$ of peak performance), and the elapsed time when using PCGE is $763 \mathrm{~s}$ (8.53\% of peak performance). As expected, the FLOP count is reduced as $6.91 \mathrm{E}+17 \rightarrow$ $3.00 \mathrm{E}+17 \rightarrow 1.76 \mathrm{E}+17$ when comparing PCGE, GAMERA, and GOJIRA, and the time-to-solution is reduced by 8.55 times and 3.67 times compared to PCGE and GAMERA, respectively. Together with the reduction in computation, we can also expect improvement in scalability with a short elapsed time, as GOJIRA reduces communication and improves load balancing. Indeed, the scalability when comparing 1,152 nodes and the full system improved from $89.9 \%$ for GAMERA and $94.2 \%$ for PCGE to $96.6 \%$. Table II shows the time and imbalance of the matrix vector computation (mostly random 
TABLE I. CONFIGURATIONS OF THE MOdELS USED TO MEASURE PERFORMANCE. MODEL SETS A AND B ARE WEAK SCALING MODEL SETS With DIFFERENT PROBLEM SIZES PER NODE, WHILE MODEL SET C IS A STRONG SCALING MODEL SET.

\begin{tabular}{|c|c|c|c|c|c|c|c|c|}
\hline model & $\begin{array}{r}\# \text { of MPI } \\
\text { processes }\end{array}$ & $\begin{array}{r}\text { \# of compute } \\
\text { cores }\end{array}$ & total DOF & $\begin{array}{l}\text { mean DOF per } \\
\text { MPI domain }\end{array}$ & $\begin{array}{r}\text { total \# of } \\
\text { tetra elements }\end{array}$ & $\begin{array}{r}\text { \# of voxel } \\
\text { elements) }\end{array}$ & $\begin{array}{r}\text { (\# of tetra } \\
\text { elements) }\end{array}$ & $\begin{array}{r}\text { \# of PCGE } \\
\text { iterations }\end{array}$ \\
\hline A-1 & 1,152 & 9,216 & $15,095,157,099$ & $13,103,435$ & $3,755,249,664$ & $449,798,144$ & $1,056,460,800$ & 1,238 \\
\hline A-2 & 2,304 & 18,432 & $30,186,410,283$ & $13,101,741$ & $7,510,474,752$ & $899,579,904$ & $2,112,995,328$ & 1,237 \\
\hline A-3 & 4,608 & 36,864 & $60,368,916,651$ & $13,100,893$ & $15,020,924,928$ & $1,799,143,424$ & $4,226,064,384$ & 1,238 \\
\hline A-4 & 9,216 & 73,728 & $120,730,026,027$ & $13,100,046$ & $30,041,800,704$ & $3,598,254,080$ & $8,452,276,224$ & 1,237 \\
\hline A-5 & 18,432 & 147,456 & $241,452,244,779$ & $13,099,623$ & $60,083,552,256$ & $7,196,475,392$ & $16,904,699,904$ & 1,238 \\
\hline A-6 & 36,864 & 294,912 & $482,888,875,563$ & $13,099,199$ & $120,167,006,208$ & $14,392,885,248$ & $33,809,694,720$ & 1,237 \\
\hline A-7 & 82,944 & 663,552 & $1,086,476,549,163$ & $13,098,917$ & $270,375,616,512$ & $32,383,893,504$ & $76,072,255,488$ & 1,236 \\
\hline B-1 & 1,152 & 9,216 & $1,620,597,627$ & $1,406,769$ & $402,571,184$ & $56,350,008$ & $64,471,136$ & 9,001 \\
\hline B-2 & 2,304 & 18,432 & $3,240,357,915$ & $1,406,405$ & $805,140,736$ & $112,700,304$ & $128,938,912$ & 8,984 \\
\hline B-3 & 4,608 & 36,864 & $6,479,049,531$ & $1,406,044$ & $1,610,280,176$ & $225,401,088$ & $257,873,648$ & 8,976 \\
\hline B-4 & 9,216 & 73,728 & $12,956,427,579$ & $1,405,862$ & $3,220,557,760$ & $450,803,328$ & $515,737,792$ & 8,970 \\
\hline B-5 & 18,432 & 147,456 & $25,909,532,667$ & $1,405,682$ & $6,441,115,328$ & $901,607,008$ & $1,031,473,280$ & 8,964 \\
\hline B-6 & 36,864 & 294,912 & $51,815,734,395$ & $1,405,592$ & $12,882,228,352$ & $1,803,213,648$ & $2,062,946,464$ & 8,960 \\
\hline B-7 & 82,944 & 663,552 & $116,577,063,003$ & $1,405,491$ & $28,985,004,864$ & $4,057,237,376$ & $4,641,580,608$ & 8,954 \\
\hline $\mathrm{C}-1$ & 1,152 & 9,216 & $12,956,427,579$ & $11,246,899$ & $3,220,557,760$ & $450,803,328$ & $515,737,792$ & 8,970 \\
\hline C-2 & 2,304 & 18,432 & & $5,623,449$ & & & & 8,971 \\
\hline $\mathrm{C}-3$ & 4,608 & 36,864 & & $2,811,725$ & & & & 8,970 \\
\hline C-4 & 9,216 & 73,728 & same as above & $1,405,862$ & same as above & same as above & same as above & 8,970 \\
\hline C-5 & 18,432 & 147,456 & & 702,931 & & & & 8,969 \\
\hline C-6 & 36,864 & 294,912 & & 351,466 & & & & 8,969 \\
\hline
\end{tabular}

access), other computation (mostly contiguous access), global communication, and peer-to-peer communication for the full system problem (model A-7). The maximum communication time (global+peer-to-peer) of all processes is at most $3.52 \mathrm{~s}$ (3.9\% of the total elapsed time) when using GOJIRA; we can see that the communication time is sufficiently small compared to the computation time ${ }^{2}$. We can see that the imbalance of elapsed time ( $\tau=\mathrm{MAX} / \mathrm{AVE})$ in "Comp. others" is large for GAMERA and PCGE; this is the major reason for the imbalance in the complete application. This imbalance is greatly improved from $\tau=2.17$ in GAMERA to $\tau=1.19$ in GOJIRA by avoiding L1 cache thrashing, which leads to an improvement in scalability for the complete application. We can see that the scalability and elapsed time are both improved by the communication-reducing algorithm and improved load balancing. With the combined improvements in single-node performance and scalability, the efficiency of application is improved from $8.6 \%$ of peak to $18.6 \%$ of peak. The efficiency of the solver becomes $18.9 \%$ of peak, corresponding to 2.01 PFLOPS, which is very high for an unstructured low-order finite element method with implicit integration.

4) Weak scaling up to 0.1T DOF on full system: Next, we measure weak scaling by fixing the problem size to 1.41 MDOF per compute node and increasing the number of nodes (model set B). Fig. 6 shows the elapsed time. The number of iterations of PCGE is similar for all models; we can see that the model set is suitable for measuring weak scaling. The smallest model (B-1 with 1.62 billion DOF) is solved in $53.5 \mathrm{~s}$ with 23.6 TFLOPS (16.0\% of peak) when using 9,216 CPU cores $(1,152$ nodes $\times 8$ CPU cores $)$, and the largest model (B-7 with 0.117 trillion DOF) is solved in $56.9 \mathrm{~s}$ with 1.60 PFLOPS (15.1\% of peak) when using the full system with $663,552 \mathrm{CPU}$ cores $(82,944$ nodes $\times 8$ CPU cores $)$. This corresponds to a 3.45-times speedup compared to GAMERA (196.7 s with performance of $8.20 \%$ of peak), and 11.4-times speedup compared to PCGE (650 s with performance of $7.82 \%$

\footnotetext{
${ }^{2}$ The time used for "Comm allreduce" is reduced from that with GAMERA using the hardware function of Tofu (Tofu Barrier Communication).
}

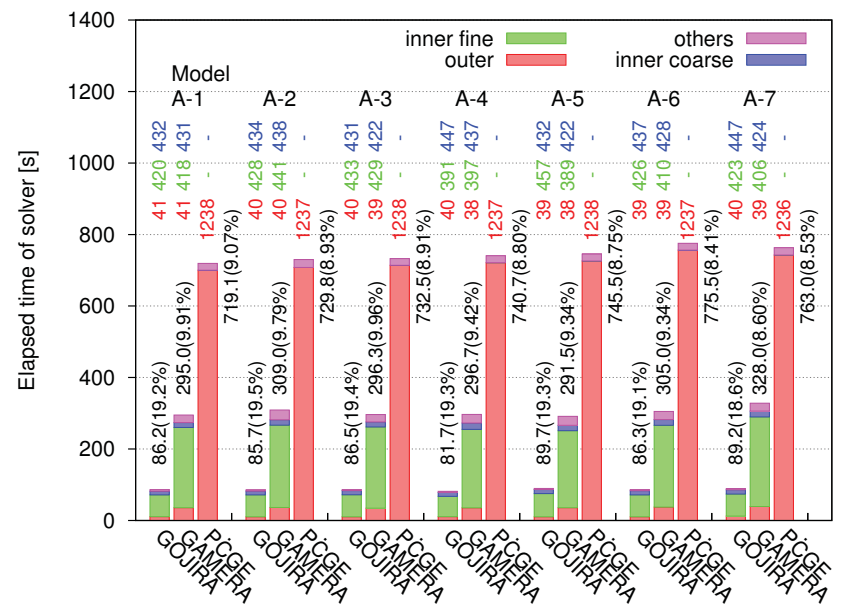

Fig. 5. Sizeup scalability for model A. The measured elapsed time for solving three time steps. Numbers in brackets indicate floating point performance, while numbers in colors indicate elapsed loops for convergence of solver (red: outer, green: inner_fine, blue: inner_coarse).

of peak). On average, GOJIRA can solve one step in $2.28 \mathrm{~s}$, which enables analysis of a practical number of steps for 0.1 TDOF problems. As it was with the 1 TDOF weak scaling problem set, the scalability (sizeup efficiency) is also improved from $91.9 \%$ of PCGE and $86.8 \%$ of GAMERA to $94.1 \%$ using GOJIRA.

5) Strong scaling: Next, we measure strong scaling. Fig. 7 shows the elapsed time with the increase in the number of CPU cores. Although there is some degradation in performance with an increase in CPU cores, we can see that good scalability is attained: the 13.0 billion DOF problem is solved in $387.4 \mathrm{~s}$ with 25.8 TFLOPS (17.52\% of peak) with 9,216 CPU cores $(1,152$ nodes $\times 8 \mathrm{CPU}$ cores, model $\mathrm{C}-1)$, in $55.4 \mathrm{~s}$ with 184.3 TFLOPS (15.62\% of peak) with 73,728 CPU cores (9,216 nodes $\times 8$ CPU cores, model $\mathrm{C}-4)$; and in $16.0 \mathrm{~s}$ with 623.8 TFLOPS (13.22\% of peak) with 294,912 CPU cores $(36,864$ nodes $\times 8 \mathrm{CPU}$ cores, model C-6). The scalability 


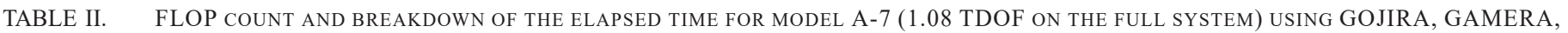
AND PCGE. NUMBERS INDICATE THE MEAN ELAPSED TIME, WHILE NUMBERS IN BRACKETS INDICATE THE MAXIMUM ELAPSED TIME AMONG ALL MPI PROCESSES. MEAN/MAX TIME WERE MEASURED BY INSERTING BARRIERS BETWEEN MEASUREMENT SECTIONS.

\begin{tabular}{|c|c|c|c|c|c|c|c|c|}
\hline method & $\begin{array}{l}\text { FLOP } \\
\text { count }\end{array}$ & $\begin{array}{l}\text { Computation } \\
\text { MATVEC (s) }\end{array}$ & $\begin{array}{r}\text { Computation } \\
\text { others (s) }\end{array}$ & $\begin{array}{r}\text { Comm. } \\
\text { allreduce (s) }\end{array}$ & $\begin{array}{r}\text { Comm. peer- } \\
\text { to-peer (s) }\end{array}$ & $\begin{array}{r}\text { Comm. pack/ } \\
\text { unpack (s) }\end{array}$ & $\begin{array}{l}\text { Total with } \\
\text { barrier (s) }\end{array}$ & $\begin{array}{r}\text { Total without } \\
\text { barrier (s) }\end{array}$ \\
\hline GOJIRA & $1.76 \mathrm{E}+17$ & $65.28(72.21)$ & $16.38(19.51)$ & $0.19(0.26)$ & $1.36(3.26)$ & $0.58(1.64)$ & 96.88 & 89.24 \\
\hline GAMERA & $3.00 \mathrm{E}+17$ & $202.2(232.3)$ & $39.45(85.43)$ & $1.12(2.31)$ & $1.31(3.45)$ & $0.69(2.50)$ & 327.3 & 328.0 \\
\hline PCGE & $6.91 \mathrm{E}+17$ & $552.37(607.5)$ & $84.71(188.0)$ & $0.91(1.38)$ & $4.17(8.82)$ & $1.97(4.19)$ & 811.2 & 763.0 \\
\hline
\end{tabular}

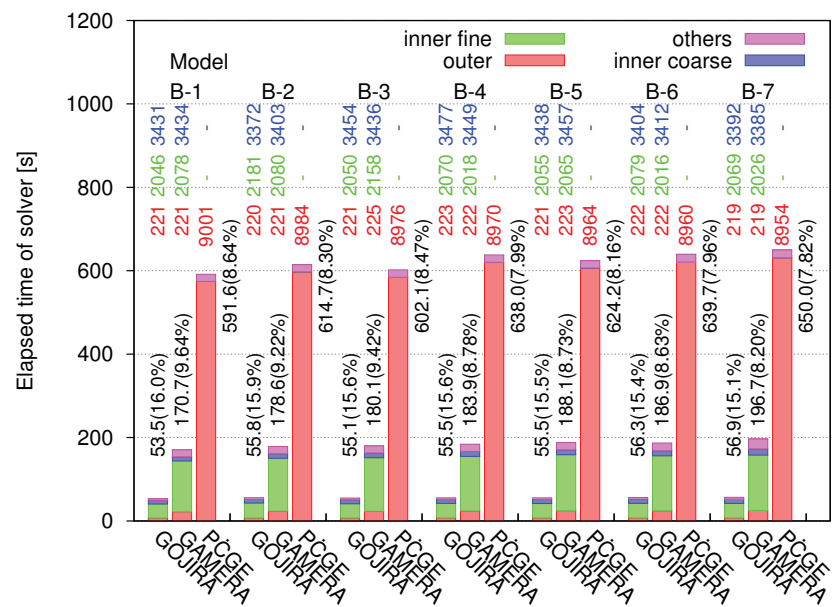

Fig. 6. Sizeup scalability for model B. The measured elapsed time for solving 25 time steps. Numbers in brackets indicate floating point performance, while numbers in colors indicate elapsed loops for convergence of solver (red: outer, green: inner_fine, blue: inner_coarse).

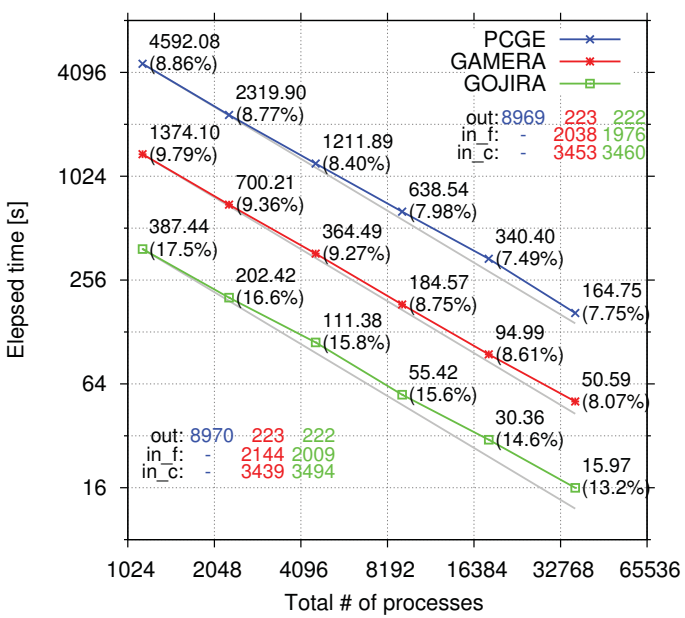

Fig. 7. Speedup scalability for model C. The measured elapsed time for solving 25 time steps. Numbers in brackets indicate floating-point performance. Black lines indicate ideal scaling. Insets (out, in_f, in_c) in bottom left and top right indicate elapsed loops of outer, inner fine and outer loops for model C-1 (1,152 processes), and model C-6 (36,864 processes), respectively.

(speedup efficiency) from 9,216 $\rightarrow 73,728$ CPU cores is $87.4 \%$, and the scalability from $9,216 \rightarrow 294,912$ CPU cores is $75.8 \%$. GAMERA has better scalability $(84.9 \%$ when from $9,216 \rightarrow 294,912$ CPU cores), because the communication time is the same with GOJIRA, but computation takes longer. We might be able to improve scalability by optimizing the computation and communication overlap using remote direct memory access (RDMA) communication.

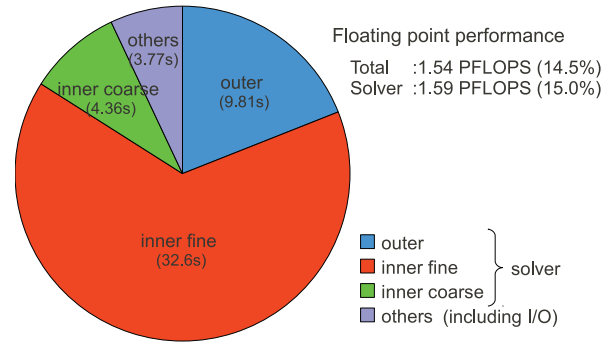

Fig. 8. Computation performance of a 0.133 TDOF application problem. Measured for the first 25 steps.

6) Performance with a practical problem: Finally, we consider the performance of the 0.133 TDOF application problem shown in Section I. Fig. 8 shows the time elapsed in the first 25 steps of the analysis. The elapsed time of the application (including I/O) is $50.6 \mathrm{~s}$; only $2.03 \mathrm{~s}$ are used to solve each step of the 0.133 TDOF dynamic nonlinear problem. Of the total processing time, $92.5 \%$ (46.8 s) of the time was taken up by the solver, showing that sufficient speedup of the solver enabled the large-scale analysis. All other operations (I/O, updating the material property with the nonlinear constitutive model, and updating the block Jacobi matrix used for the block Jacobi preconditioner) took up $7.5 \%$ of the total time, explaining the high performance of the application as a whole. The output data size of the 6,600 time step problem was $382 \mathrm{~GB}$ (surface displacement is outputted every $d t=0.01 \mathrm{~s}$ at a resolution of $2 \mathrm{~m})$. The performance is 1.59 PFLOPS $(15.0 \%$ of peak) for the solver and 1.54 PFLOPS (14.5\% of peak) for the whole application, demonstrating its high performance for a practical problem. Compared to the GAMERA application, the problem size is increased by 0.133 trillion / 10.7 billion $=12.4$ fold and the efficiency is improved by $14.5 \% / 7.59 \%=1.91$ fold. Although GAMERA could only enable an application run with 36,864 nodes, GOJIRA enables an application run using the full system by improving scalability. If the elapsed times needed for computing one second of simulation are compared, a problem 12.4 times greater in scale is computed in 0.63 times $^{3}$ the time. Although a rough estimate, this corresponds to a 7.87-fold improvement in the total time-tosolution. In actual problems, the convergence characteristics of the problem become worse for larger DOF problems. A 7.87fold improvement with larger DOF can be seen as a significant advance.

Compared to the largest-scale problem solved by state-ofthe-art systems (27 billion DOF and 6.7 billion elements with $6.93 \%$ peak performance), GOJIRA solved a problem 40 times

${ }^{3}$ (41,521 elapsed time / 30.0 simulation time; GAMERA) / (28,800 elapsed time/13.2 simulation time; GOJIRA) $=0.63$. 
larger in scale and 2.68 times better in performance efficiency (1.08 trillion DOF \& 0.270 trillion element with $18.6 \%$ peak performance) with better scalability. This represents a great improvement. With this innovation, the largest-scale stateof-the-art application using four-ninths of the $\mathrm{K}$ computer system and solving 10.8 billion DOF and 2.67 billion elements ( $7.59 \%$ of peak performance) is improved greatly to 133 billion DOF and 33.2 billion elements (14.5\% of peak performance), corresponding to a 12.4-fold increase in problem size and 1.91fold improvement in performance efficiency. Moreover, the time-to-solution, which is the most important yardstick when evaluating the performance of a practical application, is shortened by 7.87 times. By achieving performance far above the current state of the art, we have enabled the first comprehensive physics-based earthquake simulation using supercomputing.

\section{CONCLUDING REMARKS}

We developed large-scale nonlinear wave simulation code, GOJIRA, for soil amplification, and conducted a comprehensive earthquake simulation by combining it with earthquake wave propagation, structural response, and multi-agent simulation. Compared to conventional methods, we expect great improvements in estimation reliability by using physics-based comprehensive earthquake simulation. The social impact of such analyses using massive compute environments is very high. GOJIRA attains sufficient performance in massively parallel computer environments using a varying coarse/fine solution space and computation precision space, such that it suits the properties of the physical problem and computer system: a 1.08 TDOF problem was computed in $29.7 \mathrm{~s}$ per time step with 1.97 PFLOPS performance (18.6\% of peak) using the full $\mathrm{K}$ computer system with $663,552 \mathrm{CPU}$ cores, and attained a scalability (sizeup efficiency) of $96.6 \%$. Using GOJIRA, we conducted $0.133 \mathrm{TDOF}, 6.6 \mathrm{~K}$ time step, nonlinear wave propagation analysis of an actual city using $8 \mathrm{~h} 00 \mathrm{~min}$ of the full $\mathrm{K}$ computer (upper limit of the $\mathrm{K}$ computer capacity). This corresponds to $4.36 \mathrm{~s}$ per time step; we can see that superb time-to-solution was attained. Physics-based, fault-tocity-to-human earthquake simulation based on city-scale soil amplification simulation with GOJIRA should enable disaster estimates that are quantitatively different from conventional estimations. This paper proves that supercomputing can contribute to a socially important problem such as earthquake disaster mitigation.

One feature of GOJIRA is that it can compute results in double-precision accuracy using mostly single-precision computation. Although the peak performance of the $\mathrm{K}$ computer is the same regardless of whether single- or double-precision arithmetic is used, we used the $\mathrm{K}$ computer to measure performance as our target is to solve large DOF application problems on massively parallel computers. Using hardware with high single-precision arithmetic capability (e.g., Intel CPUs or GPUs), we can expect further speedup. As shown in Fig. 9, we attained 1.69 times speedup on a PC cluster with Intel CPUs when comparing mixed-precision disabled GOJIRA (only using double precision) and GOJIRA using mixed

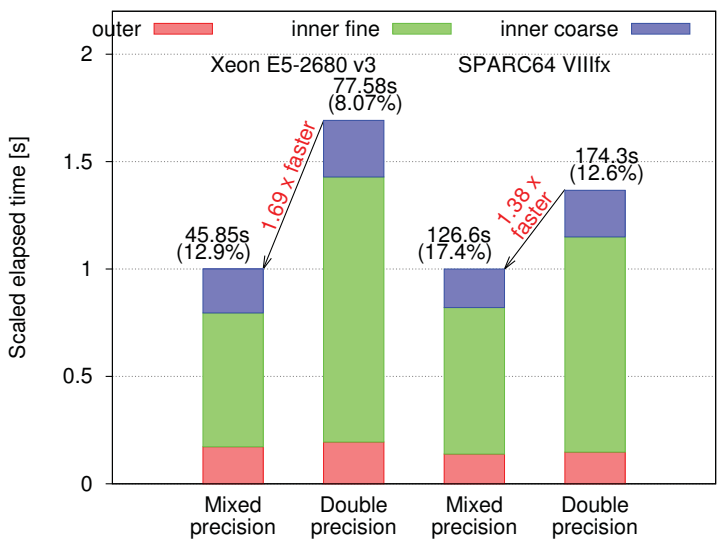

Fig. 9. Effectiveness of mixed-precision arithmetic on $\mathrm{x} 86$ and SPARC architectures. Time is scaled with the elapsed time of the mixed-precision arithmetic solver for each machine. Insets indicate the elapsed time and floating-point performance.

precision $^{4}$. As expected, the parts computed in single precision (inner fine and inner coarse) were accelerated; in particular, the inner fine part was accelerated by 1.98 times, which is near to the doubled-peak performance of single-precision arithmetic of the Intel CPU. We can see that GOJIRA effectively utilizes double-width single-precision SIMDs, which are effective in terms of both peak performance and energy efficiency, and that it is a promising method for a variety of architectures with high single-precision computation capability. From the perspective of a low-order unstructured finite element method, performance is more difficult to attain in current computers with SIMD units compared to conventional scalar CPUs due to their relatively weak random access capability. Because random access capability is expected to decrease further in the future, it is desirable to increase the number of load/store engines to increase the random access capability of the hardware, and reduce list access from the application side using a suitable algorithm. GOJIRA uses EBE with blocking to perform almost all computations by SIMD units, and reduces list access by $1 / 2.22$ of the standard finite element method. Together with the reduction in communication, the effectiveness of GOJIRA should increase when using future machines, such as next-generation Intel machines or a post-K computer, which is expected to have longer SIMD units, but relatively less communication capability.

Urban-scale soil amplification simulation, which is difficult with conventional methods due to the huge computational cost, is a simpler problem with the advent of GOJIRA. Needless to say, the scientific rationality of earthquake disaster estimates will be improved. Furthermore, a broad ripple effect involving other problems related to earthquake disasters is expected. For example, the huge tsunami induced by the Tohoku Earthquake in 2011 caused catastrophic damage. The application of GOJIRA to crust deformation analysis used for tsunami source estimation should improve estimations of such tsunamis. Linear wave propagation analysis is used for

\footnotetext{
${ }^{4}$ We used a PC cluster consisting of 18 compute nodes, each with two Xeon E5-2680 v3 CPUs and 36 nodes of the K computer. Twelve OpenMP threads were used per CPU for the PC cluster and eight OpenMP threads were used for each node of the $\mathrm{K}$ computer. The problem size was set to $1,313,659$ $\mathrm{DOF} /$ process, which is similar to the weak scaling model set B in Table I, and attaches one MPI process per CPU.
} 
earthquake wave propagation analyses (e.g., SeisSol), but some researchers have pointed out that the phenomenon becomes partly nonlinear in the case of large earthquakes. The application of GOJIRA to such wave propagation problems is also expected. In addition to all of these application possibilities, the development of GOJIRA itself has shifted the frontier of earthquake disaster estimation toward the social/economic response by demonstrating the possibility of simulation from the earthquake source to the human response using highprecision physics-based simulations. Such computations have greater computation costs, but should be a fascinating topic enabling supercomputing to contribute to society; we are eager to tackle this challenging problem. Let's step forward to the new frontier of supercomputing.

\section{ACKNOWLEDGMENTS}

Results are obtained using the $\mathrm{K}$ computer at the RIKEN Advanced Institute for Computational Science (Proposal number hp140223, hp150217, hp150175, hp150127). We used KiK-net and Japan Seismic Hazard Information Station of National Research Institute for Earth Science and Disaster Prevention (NIED), and National Digital Soil Map provided by Japanese Geotechnical Society. This work was supported by JSPS KAKENHI Grant Numbers 25220908, 25630192, 26249066, 12J08183, 15K18110 and flagship 2020 project (priority issues 3). This work is partially funded by MEXT's program for the Development and Improvement for the Next Generation Ultra High-Speed Computer System, under its Subsidies for Operating the Specific Advanced Large Research Facilities.

\section{REFERENCES}

[1] P. Somerville, N. Collins, N. Abrahamson, R. Graves, and C. Saikia "GROUND MOTION ATTENUATION RELATIONS FOR THE CENTRAL AND EASTERN UNITED STATES," Final Report (June 30, 2001). [Online].

http://earthquake.usgs.gov/hazards/products/conterminous/2008/ 99HQGR0098.pdf

[2] Second report of the Nankai Trough Large Earthquake Model Committee, Cabinet Office, Government of Japan (August 28,2012). [Online]. http://www.bousai.go.jp/jishin/nankai/model/index.html

[3] Disaster assessment of Tokyo due to large earthquakes such as the Nankai Trough Earthquake, Tokyo Metropolitan Government (May 14, 2013). [Online].

http://www.bousai.metro.tokyo.jp/taisaku/1000902/1000402.html

[4] H. Miyazaki, Y. Kusano, N. Shinjou, F. Shoji, M. Yokokawa and T. Watanabe. "Overview of the K computer system," FUJITSU Sci. Tech. J., 2012, Vol. 48, No. 3, pp.302-309.

[5] T. Tiankai, Y. Hongfeng, L. Ramirez-Guzman, J. Bielak, O. Ghattas, M. Kwan-Liu, and D. R. O'Hallaron, "From mesh generation to scientific visualization: an end-to-end approach to parallel supercomputing," Proceedings of the 2006 ACM/IEEE conference on Supercomputing (SC'06). ACM, New York, NY, USA, 2006, Article 91 DOI $=10.1145 / 1188455.1188551$

[6] C. Yifeng Cui, K. B. Olsen, T. H. Jordan, K. Lee, J. Zhou, P. Small, D. Roten, G. Ely, D. K. Panda, A. Chourasia, J. Levesque, S. M. Day and P. Maechling, "Scalable Earthquake Simulation on Petascale Supercomputers," Proceedings of the 2010 ACM/IEEE International Conference for High Performance Computing, Networking, Storage and Analysis (SC'10). IEEE Computer Society, Washington, DC, USA, 2010, pp.120. DOI=10.1109/SC.2010.45 http://dx.doi.org/10.1109/SC.2010.45
[7] M. Rietmann, P. Messmer, T. Nissen-Meyer, D. Peter, P. Basini, D Komatitsch, O. Schenk, J. Tromp, L. Boschi, and D. Giardini. "Forward and adjoint simulations of seismic wave propagation on emerging largescale GPU architectures," Proceedings of the International Conference on High Performance Computing, Networking, Storage and Analysis (SC'12). IEEE Computer Society Press, Los Alamitos, CA, USA, 2012, Article 38, 11 pages.

[8] Y. Cui, E. Poyraz, K. B. Olsen, J. Zhou, K. Withers, S. Callaghan, J. Larkin, C. Guest, D. Choi, A. Chourasia, Z. Shi, S. M. Day, P. J. Maechling and T.H. Jordan. "Physics-based seismic hazard analysis on petascale heterogeneous supercomputers," Proceedings of the International Conference on High Performance Computing, Networking, Storage and Analysis, (SC'13). IEEE Computer Society Press, New York, NY, USA, 2013, Article 70, 12 pages.

[9] A. Heinecke, A. Breuer, S. Rettenberger, M. Bader, A-A. Gabriel, C. Pelties, A. Bode, W. Barth, X-K. Liao, K. Vaidyanathan, M. Smelyanskiy, P. Dubey, "Petascale High Order Dynamic Rupture Earthquake Simulations on Heterogeneous Supercomputers," Proceedings of the International Conference on High Performance Computing, Networking, Storage and Analysis, (SC'14), pp 3-14, 2014.

[10] Japan Seismic Hazard Information Station, National Research Institute for Earth Science and Disaster Prevention. [Online]. http://www.j-shis.bosai.go.jp/en/

[11] Report of the Assumed Tokyo Large Earthquake Model Committee, Cabinet Office, Government of Japan (December 28, 2013). [Online]. http://www.bousai.go.jp/kaigirep/chuobou/senmon/shutochokkajishinmodel/

[12] P. E. B. Quinay, T. Ichimura, M. Hori, A. Nishida, S. Yoshimura. "Seismic Structural Response Estimates of a High-Fidelity Fault-Structure System Model Using Multiscale Analysis with Parallel Simulation of Seismic Wave Propagation," Bulletin of the Seismological Society of America, 103, pp. 2094-2110 2013.

[13] R. Taborda and J. Bielak. "Large-scale earthquake simulation: computational seismology and complex engineering systems," Computing in Science \& Engineering, 13, pp. 14-27, 2011.

[14] T. Ichimura, K. Fujita, M. Hori, T. Sakanoue and R. Hamanaka. "Threedimensional nonlinear seismic ground response analysis of local site effects for estimating seismic behavior of buried pipelines," Journal of Pressure Vessel Technology, American Society of Mechanical Engineers, 136, paperID 041702, 2014.

[15] K. Fujita, T. Ichimura, M. Hori, L. Maddegedara and S. Tanaka "Scalable multicase urban earthquake simulation method for stochastic earthquake disaster estimation," Procedia Computer Science, 2015 (accepted)

[16] T. Ichimura, K. Fujita, S. Tanaka, M. Hori, M. Lalith, Y. Shizawa, and H. Kobayashi. "Physics-based urban earthquake simulation enhanced by 10.7 BlnDOF x $30 \mathrm{~K}$ time-step unstructured FE non-linear seismic wave simulation," Proceedings of the International Conference on High Performance Computing, Networking, Storage and Analysis, (SC'14), pp 15-26, 2014.

[17] National Digital Soil Map, The Japanese Geotechincal Society, [Online]. http://www.denshi-jiban.jp/

[18] Strong-motion seismograph networks (K-NET, KiK-net), National Research Institute for Earth Science and Disaster Prevention, [Online]. http://www.kyoshin.bosai.go.jp/

[19] K. Zia, A. Riener, K. Farrahi and A. Ferscha. "A New Opportunity to Urban Evacuation Analysis: Very Large Scale Simulations of Social Agent Systems in Repast HPC," ACM/IEEE/SCS 26th Workshop on Principles of Advanced and Distributed Simulation, 2012.

[20] M. L. L. Wijerathne, M. Hori, T. Ichimura, S. Tanaka. "Parallel Scalability Enhancements of Seismic Response and Evacuation Simulations of Integrated Earthquake Simulator," High Performance Computing for Computational Science - VECPAR 2012 , Lecture Notes in Computer Science, Vol. 7851, 2013, pp 105-117, 2013.

[21] M. L. Wijerathne, L. A. Melgar, M. Hori, T. Ichimura, and S. Tanaka. "HPC enhanced large urban area evacuation simulations with vision based autonomously navigating multiagents," Procedia Computer Science, pp 1515-1524, 2013.

[22] L. E. A. Melgar, M. Lalith, M. Hori, T. Ichimura, S. Tanaka. "A Scalable Workbench for Large Urban Area Simulations, Comprised of Resources for Behavioural Models," Interactions and Dynamic Environments, 
PRIMA 2014: Principles and Practice of Multi-Agent Systems, Lecture Notes in Computer Science, Vol. 8861, pp 166-181, 2014.

[23] I.M. Idriss, R.D. Singh and R. Dobry. "Nonlinear behavior of soft clays during cyclic loading," Journal of the Geotechnical Engineering Division, 1978, 104, pp. 1427-1447.

[24] G. Masing. "Eigenspannungen und verfestigung beim messing," Proceedings of the 2nd International Congress of Applied Mechanics, 1926, pp. 332-335 (in German).

[25] M. Ogino, R. Shioya and H. Kanayama. "An inexact balancing preconditioner for large-scale structural analysis," Journal of Conputational Science and Technology, 2008, 2(1), pp. 150-161.

[26] H. Kawai, M. Ogino, R. Shioya and S. Yoshimura. "Large-scale elastplastic analysis using domain decomposition method optimized for multi-core CPU architecture," Key Engineering Materials, 2011, 462463, pp. 605-610.

[27] H. Akiba, T. Ohyama, Y. Shibata, K. Yuyama, Y. Katai, R. Takeuchi, T. Hoshino, S. Yoshimura, H. Noguchi, M. Gupta, J. Gunnels, V. Austel, Y. Sabharwal, R. Garg, S. Kato, T. Kawakami, S. Todokoro, J. Ikeda. "Large scale drop impact analysis of mobile phone using ADVC on Blue Gene/L," Proceedings of the 2006 ACM/IEEE conference on Supercomputing (SC'06), 2006, ACM, New York, NY, USA, Article 46. $\mathrm{DOI}=10.1145 / 1188455.1188503$

[28] J. Mandel, "Balancing domain decomposition," Communications in numerical methods in engineering, 1993, Volume 9, Issue 3, pp.233241.

[29] Earth Simulator (ES), http://www.jamstec.go.jp/es/en/es1/index.html

[30] H. Bao, J. Bielak, O. Ghattas, D.R. O'Hallaron, L.F. Kallivokas, J.R. Shewchuk, J. Xu, "Earthquake ground motion modeling on parallel computers," in Proceedings of the 1996 ACM/IEEE conference on Supercomputing, 1996.

[31] T. Ichimura, M. Hori, H. Kuwamoto, Earthquake Motion Simulation with Multi-Scale Finite Element Analysis on Hybrid Grid, Bulletin of the Seismological Society of America, 97, pp. 1133-1143, 2007.

[32] T. Ichimura, M. Hori, J. Bielak. "A Hybrid multiresolution meshing technique for finite element three-dimensional earthquake ground motion modeling in basins including topography," Geophysical Journal International, 2009, 177, pp. 1221-1232.

[33] G. H. Golub, Q. Ye Inexact conjugate gradient method with inner-outer iteration. SIAM., Journal on Scientific Computing 1997; 21(4):13051320.

[34] Y. Saad, 2003, Iterative methods for sparse linear systems (2nd ed.), SIAM.

[35] J. M. Winget, T. J. R. Hughes "Solution algorithms for nonlinear transient heat conduction analysis employing element-by- element iterative strategies," Computer Methods in Applied Mechanics and Engineering 1985; 52:711-815.

[36] O.C. Zienkiewicz, R. L. Taylor. The Finite Element Method for Solid and Structural Mechanics (6th ed.), 2005, Elsevier.

[37] METIS 5.1.0, [Online]. http://glaros.dtc.umn.edu/gkhome/metis/metis/overview

[38] E. Hairer, S. P. Nørsett, G. Wanner, 1993, Solving ordinary differential equations I: Non stiff problems (2nd ed.), Springer.

[39] Y. Ajima, T. Inoue, S. Hiramoto and T. Shimizu. "Tofu: interconnect for the K computer," FUJITSU Sci. Tech. J., 2012, Vol.48, No.3, pp.280285.

[40] OpenMPI, [Online]. http://www.open-mpi.org/

[41] Strong ground motion of The Southern Hyogo prefecture earthquake in 1995 observed at Kobe JMA observatory, Japan Meteorological Agency, [Online]. http://www.data.jma.go.jp/svd/eqev/data/kyoshin/jishin/hyogo_nanbu/ dat/H1171931.csv 